\title{
Guest editorial for special section on a new culture of learning: Developing computing in the curriculum and advancing digital pedagogy
}

\author{
Andrej Brodnik ${ }^{1,2} \cdot$ Cathy Lewin $^{3}$
}

Published online: 3 February 2017

(C) Springer Science+Business Media New York 2017

This special issue of EAIT brings a selection of articles on the topic "A New Culture of Learning: Computing and Next Generations" (Brodnik and Lewin 2015). After a thorough consideration and further review process, eight papers were selected for inclusion in this issue and subsequently developed further by the authors. They can be divided into two broad groups: papers on Computer Science Education Research (CSER) and papers on the use of ICT in education.

There is a growing interest in the field of CSER from academics, policy makers and practitioners, and we are delighted to present three papers which will make a significant contribution to this field. The reason for this interest is a worldwide understanding that we need to teach students to become creators and not mere users (consumers) of ICT (IFIP WCCE, Torun 2013). One of the most widely cited sources on the urgency of change in CS education is the report "Shutdown or Restart?" published by The Royal Society in the UK in 2012. The papers we include in this special issue in some sense also originate from this report.

The first paper in this special issue from Don Passey focuses on the need to balance computer science and 'ICT' in the curriculum. In this subsequent paper, he provides a rationale for incorporating computer science in school curricula, drawing on policy analysis. This is a timely point at which to craft a positional paper on the role of

Cathy Lewin

c.lewin@mmu.ac.uk

Andrej Brodnik

andrej.brodnik@fri.uni-1j.si

1 Faculty of Computer and Information Science, University of Ljubljana, Ljubljana, Slovenia

2 Department of Information Science and Technology, University of Primorska, Koper, Slovenia

3 Education and Social Research Institute, Faculty of Education, Manchester Metropolitan University, Manchester, UK 
computer science in (compulsory) education, given the increasing interest at international level and the current lack of research in this field. Having presented six arguments for incorporating computer science in the school curriculum, he teases out the implications of achieving this for schools, teachers and learners, cautioning that much needs to be done to reverse the declining interest of young people in undertaking academic qualifications in computer science and other related subjects. Given the current lack of research in this area, he concludes with recommendations for further studies which could contribute to a deeper understanding of how the six arguments presented might play out in school contexts.

In the next contribution Mary Webb, Niki Davis, Tim Bell, Yaacov J. Katz, Nicholas Reynolds, Dianne P. Chambers and Maciej M. Syslo present an overview of CS curriculum development in the last few years. The paper is based on discussions within the IFIP Education Community and on analyses of CS curricula from five different countries world-wide. Through the analyses the authors justify the importance of CS as so called "powerful knowledge". Furthermore, they also identify areas of both broad consensus and issues of controversy in CS education. For example, they identify a strong consensus that starting learning CS early in primary school reaps opportunities and benefits, with one of the benefits being equity among the learners. However, they also identify teachers' professional development as a major risk in CS education. The paper also discusses topics included in curricula, and how and when they are introduced. Indeed, due to its comparative nature the paper will probably become a good reference point to reflect on in future studies.

The last paper on CS education, by Sue Sentance and Andrew Csizmadia, turns the table and considers the teacher's perspective. The authors argue, quite rightly, that the shift from teaching ICT to CS requires teachers to develop new subject knowledge and new pedagogical approaches. They conducted a survey, which more than 300 teachers responded to offering their opinions of current challenges and effective teaching strategies. As well as teachers' subject knowledge requiring development, the teachers reported that students need to adjust, particularly in relation to the emphasis on problem solving which they are not necessarily accustomed to. Sentance and Csizmadia sensibly recommend that teachers need more training but also that more allowances need to be made initially for students to get used to the new subject material and approaches. The authors conclude that teachers should focus on developing students' skills in problem solving and computational thinking, and helping their students to learn to become more resilient.

The second collection of papers reflect a broad range of interests in the use of ICT to support teaching and learning, four concerning K-12 and one relating to the higher education sector. The first two papers in this group illustrate the continued interest in the role of games, gamification and ludicization in school classrooms to improve learner motivation. The final three papers highlight the impact of $1: 1$ provision on students' literacy skills, present an international comparison of factors affecting teachers' uptake of ICT in the classroom, and propose a framework for creative online collaboration in higher education.

The first of two gamification papers is by Eric Sanchez, Shawn Young, and Caroline Jouneau-Sion and is built around the Classcraft role-playing game. The game was developed for classroom management in a high school. In the game, a 
teacher creates teams consisting of students with different roles and teams have to function in a collaborative way. In some sense, a team is a very small class where students have to collaborate to make an improvement. The authors present the results of experiments that took place in France and Quebec respectively. Both experiments showed an improved motivation of students for engaging in learning. Moreover, improvement was also seen in the area of mutual help. The authors justify the change from 'gamification' to 'ludicization' as Classcraft was not only used for its gamification elements (such as points or rewards), but also as a reflexive space for metaphorizing the real life situations - like helping each other, or, in general, building an inter-personal relationship.

Sónia Cruz, Ana Amélia A. Carvalho and In s Araújo present a theoreticallygrounded design for a mobile game to teach Portuguese history. Through conducting a review of games for learning history, the authors argue that game-based learning can stimulate curiosity in history warranting its use in school classrooms. They also conclude that games should be designed to be integrated in formal teaching with supporting resources rather than to be a supplementary activity. They move on to discuss findings from a survey of young people's game playing preferences in order to develop a game with features that students will find appealing and engaging. The authors reveal that aesthetics and opportunities for progression are important for both boys and girls, and confirm findings from the literature that boys prefer adventure, action and sport game genres while girls prefer simulations that promote caring for example. The games that young people suggest that they play most frequently are then analysed in relation to Gee's learning principles, identifying 8 of the 32 which commonly occur in games that young people choose to play for their own interest. In conclusion, the authors present the design of a mobile game for teaching Portuguese history, drawing on the findings of the literature review, survey and analysis of commonly played games.

The last group of papers starts with a paper authored by Bent B. Andresen, in which he discusses the acquisition of literacy skills in a 1:1 classroom. Since he is predominantly interested in the impact of the 1:1 classroom on students, he defines it solely as a classroom where each student has an e-device. His study was conducted in the first, third, seventh and eighth grades of Danish schools. Although the study was undertaken at a national level it presents some interesting challenges of a broader interest. For example, the paper points out that despite governmental effort $60 \%$ of teachers need more knowledge on how to use digital tools to support teaching and learning. On the other hand, the technology facilitated an efficient motivational moment for students as they were spending more time accessing the multimodal learning environment. This led to an improvement in their digital literacy skills - reading, creating and sharing of learning material. This result actually contradicts internationally comparative analyses run by the OECD and hence opens an interesting question as to why this is the case.

The study in the next paper is based on the ICIL 2013 framework, in which the students' computer and information literacy is influenced by antecedents and processes at different levels (personal, home, classroom, and the wider community). For example, the classroom (school) level process includes ICT based teaching and consequently in-class use of ICT. Kerstin Drossel, Birgit Eikelmann and Julia Gerick study the characteristics of schools and teachers that can be used to predict the frequency of in-class ICT use. They draw on data from more than 8000 teachers in 
The Netherlands, Denmark, Australia, Poland, and Germany. The characteristics they considered were related to schools, the teacher's attitude toward use of ICT, school and teaching process, and the teacher's background. Although the paper presents more detailed results, we reveal two here to stimulate your curiosity to read the whole contribution. For example, they discovered that there are more countryspecific characteristics than similarities. However, the characteristic of teachers' attitudes toward educational use of ICT emerged as the one with the most impact across the studied countries.

Finally, Bjoern Stockleben, Martyn Thayne, Seija Jäminki, Ilkka Haukijärvi, Nicholas Mavengere, Muhammet Demirbilek and Miko Ruohonen report on the first phases of the OnCreate project which seeks to contribute to knowledge about the best ways to facilitate collaborative work online in creative disciplines in the higher education sector. Arguing that collaboration is a key skill demanded of professionals in the creative sector (and beyond), the authors draw on a review of literature to identify the characteristics of creative processes and highlight the potential challenges of supporting these in an online collaborative environment together with contextual factors for design of such online services. They then apply the derived framework, consisting of the identified key challenges and contextual key factors, to case studies of best practice from the 10 consortium members. Finally, the authors put the good practice principles to work in the design of an online course involving four participating universities. They conclude with recommendations for applying the framework in future developments of online collaborative courses and suggestions for future research.

We hope that you find this collection of papers thought provoking and that either individually or collectively they serve to inform your future research activities. We would like to thank the reviewers of all the papers considered for this special issue for taking the time to provide detailed feedback for the authors. We would also like to thank the authors themselves for responding promptly and fully to our requests for revisions.

\section{References}

Brodnik, A. \& Lewin, C. (Eds.) (2015). IFIP TC3 Working Conference A New Culture of Learning: Computing and next Generations: Proceedings. July 1st - July 3rd, 2015, Vilnius, Lithuania. Vilnius: Vilnius University. http://www.ifip2015.mii.vu.lt/file/repository/IFIP_Proceedings.pdf. Accessed 30 January 2017. 